\title{
Asymmetric Photolysis of 2-Phenylcycloalkanones with Circularly Polarized Light: A Kinetic Model for Magnetic Field Effects
}

\author{
Shigeru Kohtani, ${ }^{*}$ Masahide Sugiyama, Yoshihisa Fujiwara, ${ }^{\dagger}$ Yoshifumi Tanimoto, $^{\dagger}$ and \\ Ryoichi Nakagaki $i^{\dagger \dagger}$ \\ Graduate School of Natural Science and Technology, Kanazawa University, Takara-machi, Kanazawa 920-0934 \\ †Graduate School of Science, Hiroshima University, Kagamiyama, Higashi-Hiroshima 739-8526 \\ $\dagger †$ Faculty of Pharmaceutical Sciences, Kanazawa University, Takara-machi Kanazawa 920-0934
}

(Received September 4, 2001)

\begin{abstract}
Magnetic field effects (MFE) on asymmetric photolysis involving a biradical intermediate have been investigated on a kinetic model where an intersystem crossing (ISC) of the intermediate is taken into account. Changes in the enantiomeric excesses (ee) of chiral substances with circularly polarized light (CPL) irradiation have been simulated, and the necessary conditions for observing the MFE were obtained. The asymmetric photolysis of racemic 2-phenylcycloalkanones (2-PCAs) with CPL has been carried out in both the presence and absence of a magnetic field. Since the anisotropy $g$ factors of 2-PCAs are considerably large, the CPL-induced ee are achieved to a few percent after $90 \%$ decomposition though the MFE are not observed. The photolysis mechanism of 2-PCAs in an air-saturated solution has been also clarified. Triplet acyl-benzyl biradicals, formed via photochemical $\alpha$-cleavage of 2-PCAs, react with $\mathrm{O}_{2}$ dissolved in the solution and result in the formation of acetophenone and alkenoic acids. The bimolecular reactions are diffusion-controlled and the rates are comparable to those of ISC to the singlet biradicals for all 2-PCAs. The recombination yields of the biradicals are sufficiently large. However, the biradical ISC rate shows little magnetic field dependence, which explains the absence of the MFE in this asymmetric photolysis.
\end{abstract}

Absolute asymmetric synthesis is a methodology used for asymmetric induction under physical forces, and has been attracted much attention in relation to the origin of biomolecular homochirality. ${ }^{1-3}$ Among the physical forces, circularly polarized light (CPL) is the most extensively studied and well documented chiral source. ${ }^{1-5}$ Enantiomeric excess (ee) induced by CPL irradiation is brought about through the preferential excitation of one of enantiomers in a racemic mixture because of circular dichroism of chiral substances. Kinetic studies of various type of asymmetric photoreactions with CPL have been carried out both theoretically and experimentally. ${ }^{4-9}$ On the contrary, the role of a magnetic field for absolute asymmetric synthesis has been controversial for a long time., ${ }^{2,3}$ Recently, Rikken and Raupach successfully demonstrated an enantioselective photo-deracemization of chiral tris[ethanedioato(2-)$\left.O, O^{\prime}\right] \mathrm{Cr}(\mathrm{III})$ complex by applying a static magnetic field either parallel or anti-parallel to the propagation direction of light, though the observed ee was extremely small (ca. $10^{-2} \%$ ee at $10 \mathrm{~T}$ ) compared to that achieved by CPL irradiation. ${ }^{10}$

On the other hand, it has been known that a magnetic field affects chemical reactions involving radical pair or biradical intermediates where the rate of an intersystem crossing (ISC) of the intermediates is influenced by a magnetic field. ${ }^{11-17}$ End-product yields in some photochemical reactions of bifunctional chain molecules are also altered via a magnetic-field-dependent spin-conversion process of the intermediates. ${ }^{18-20}$ These magnetic field effects (MFE) are closely related to spin dynamics in which singlet-triplet state mixing is induced by hyperfine coupling and electronic Zeeman mechanism.

There have been two notable studies concerning MFE on asymmetric reactions and the radical pair mechanism. First, Lem and Turro reported enantioselective radical pair recombination in the photolysis of meso- or dl-2,4-diphenylpentan-3one within a supercage of zeolites which was modified with a chiral inductor (diethyl L-tartrate or ephedrine). ${ }^{21}$ They claimed that when the carbon atom at the ketone is replaced by ${ }^{13} \mathrm{C}$, the enantioselectivity is enhanced by increasing the ISC rate in an $\alpha$-methylbenzyl and ( $\alpha$-methylbenzyl)acyl radical pair generated in the supercage, though external MFE were not observed. Second, Hegstrom and Kondepudi have predicted dramatic MFE in a chirally autocatalytic reaction involving the ISC process of radical pairs from a computational kinetic simulation. $^{22}$ However, it seems to be difficult to demonstrate their prediction because the chirally autocatalytic reaction is presently a rare phenomenon. ${ }^{23,24}$

Our strategy to obtain large MFE in asymmetric reactions is that the magnetic-field-dependent ISC process of radical pairs or biradicals is incorporated into asymmetric photolysis with CPL. While CPL is the only chiral source, the magnetic field has no chirally asymmetric influence. ${ }^{2,3}$ However, the magnetic field can change the ee of substances kinetically by affecting the ISC rate. In this paper, we propose a kinetic model involving the ISC process, and discuss our simulated ee changes in initially racemic substances with CPL irradiation in order to understand the conditions for observing MFE. We then describe experimental attempts to observe the MFE in an asym- 


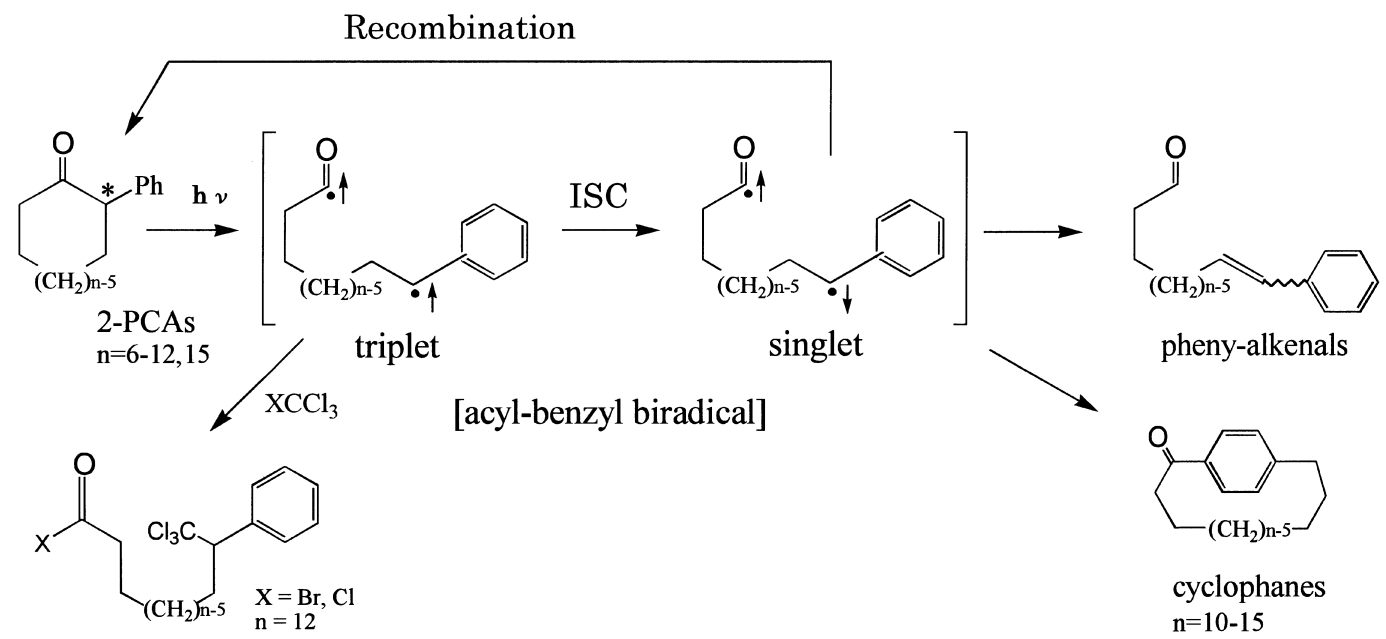

Scheme 1. Photolysis mechanism of 2-phenylcycloalkanones (2-PCAs, $n=6-12,15$ ).

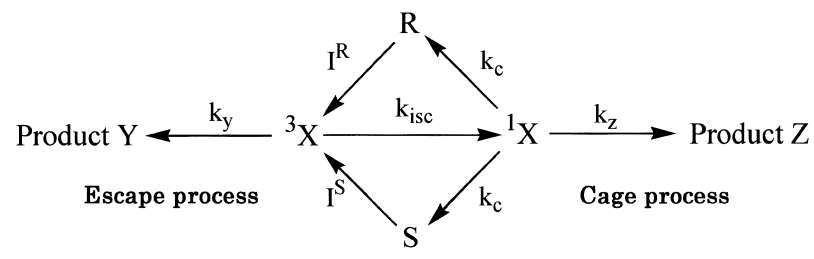

Scheme 2. Schematic diagram of asymmetric photolysis involving ISC process of radical pairs or biradicals $\left({ }^{3} \mathrm{X}\right.$ : triplet, ${ }^{1} \mathrm{X}$ : singlet). The intersystem crossing rate $\left(k_{\text {isc }}\right)$ depends on magnetic field strengths.

metric photolysis of 2-phenylcycloalkanones (2-PCAs, $n=6-$ 8 ) induced by CPL irradiation. The reasons for selecting this reaction system are as follows: (1) 2-PCAs are expected to have a large Kuhn anisotropy $g$ factor because of an asymmetric carbon next to the carbonyl group in the cycloalkanone ring. ${ }^{4,5}$ In general, chiral cyclic ketones, such as 2-PCAs, have a large anisotropy $g$ factor because the $n \pi^{*}$ transition of carbonyl group is electronically forbidden, but magnetically allowed. ${ }^{25}$ (2) The photolysis of 2-PCAs involves the ISC process of acyl-benzyl biradical as shown in Scheme 1, which has been clarified by chemically induced dynamic nuclear polarization (CIDNP), ${ }^{26-28}$ laser flash photolysis, ${ }^{29-33}$ and photoproducts analyses. ${ }^{34-38}$ (3) Magnetic-field-dependent CIDNP and MFE on the ISC rates of acyl-benzyl biradicals are observed in the photolysis of small $(n=6-8)$ and large $(n=10$ 12) ring size 2-PCAs. ${ }^{28,31-33}$ We further investigated the photolysis mechanism of 2-PCAs in air-saturated solutions by using a GC/MS photoproduct analyses, transient absorption measurements, and by examining the time evolution of the enantiomer concentrations for the starting species based on our kinetic simulation. The necessary conditions for observing large MFE derived from the kinetic simulation are compared with the experimental results obtained in this work. Finally, the magnetic field control of photoinduced ee is discussed in some detail.

\section{Kinetic Simulation}

In asymmetric photolyses with CPL, one of both enantiomers is preferentially excited and subsequent decomposition and/or isomerization reaction proceed upon left- or right-CPL irradiation. Therefore, enantiomeric enrichment for another enantiomer is achieved. The degrees of the preferential excitation is defined by the Kuhn anisotropy $g$ factor, $\left(\varepsilon_{\mathrm{L}}-\varepsilon_{\mathrm{R}}\right) / \varepsilon=$ $\Delta \varepsilon / \varepsilon$, where $\varepsilon=\left(\varepsilon_{\mathrm{L}}+\varepsilon_{\mathrm{R}}\right) / 2$. This factor determines ee at a certain extent of decomposition upon monochromatic CPL irradiation. ${ }^{4-9}$

Scheme 2 illustrates a kinetic model for asymmetric photolysis involving the ISC of radical pair or biradical intermediates. This reaction scheme is based on a photochemical reaction of 2-PCAs in Scheme 1. The photocleavage of a chiral compound ( $R$ and $S$ enantiomer) takes place in the excited triplet state, thereby resulting in the formation of a triplet biradical $\left({ }^{3} \mathrm{X}\right)$. The initially prepared triplet biradical undergoes two competitive processes, namely, an escape process to Product $\mathrm{Y}$ and ISC to a singlet biradical $\left({ }^{1} \mathrm{X}\right)$. Finally, recombination and cage product formation from ${ }^{1} \mathrm{X}$ take place to yield the starting $R$ and $S$ enantiomers with equal rate constants $\left(k_{\mathrm{c}}\right)$ and Product $\mathrm{Z}$, respectively. The formation rates of ${ }^{3} \mathrm{X}$ from the starting $R$ and $S$ enantiomer are different upon CPL irradiation as described below: ${ }^{4,8}$

$$
\begin{aligned}
& I^{R}=1000 I_{0} \eta\left[\frac{\left(1-10^{-A}\right)}{A} \varepsilon_{R}[R]\right] \\
& I^{S}=1000 I_{0} \eta\left[\frac{\left(1-10^{-A}\right)}{A} \varepsilon_{S}[S]\right]
\end{aligned}
$$

where $I_{0}$ is the incident light intensity (einstein $/ \mathrm{s}^{\bullet} \mathrm{cm}^{2}$ ). $\varepsilon_{R}$ and $\varepsilon_{S}$ are the molar extinction coefficients for $R$ and $S$ enantiomers toward left- or right-CPL at the irradiation wavelength, respectively. $A$ is absorbance $\left(\varepsilon_{R}[R]+\varepsilon_{S}[S]\right)$, and $\eta$ is the quantum yield of ${ }^{3} \mathrm{X}$ from the excited starting material. Kinetic equations for the concentration changes in $R, S,{ }^{3} \mathrm{X}$, and ${ }^{1} \mathrm{X}$ are described as follows (see Scehme 2):

$$
\begin{aligned}
& \frac{\mathrm{d}[R]}{\mathrm{d} t}=-I^{R}+k_{\mathrm{c}}\left[{ }^{1} \mathrm{X}\right] \\
& \frac{\mathrm{d}[S]}{\mathrm{d} t}=-I^{S}+k_{\mathrm{c}}\left[{ }^{1} \mathrm{X}\right]
\end{aligned}
$$




$$
\begin{aligned}
& \frac{\mathrm{d}\left[{ }^{3} \mathrm{X}\right]}{\mathrm{d} t}=I^{R}+I^{S}-\left(k_{i s c}+k_{y}\right)\left[{ }^{3} \mathrm{X}\right] \\
& \frac{\mathrm{d}\left[{ }^{1} \mathrm{X}\right]}{\mathrm{d} t}=k_{i s c}\left[{ }^{3} \mathrm{X}\right]-\left(2 k_{\mathrm{c}}+k_{\mathrm{z}}\right)\left[{ }^{1} \mathrm{X}\right] .
\end{aligned}
$$

Assuming that the concentration changes in ${ }^{3} \mathrm{X}$ and ${ }^{1} \mathrm{X}$ are negligible in a steady-state approximation, i.e. $\mathrm{d}\left[{ }^{3} \mathrm{X}\right] / \mathrm{d} t=0$ and $\mathrm{d}\left[{ }^{1} \mathrm{X}\right] / \mathrm{d} t=0$, the differential equations for $[R]$ and $[S]$ are derived as follows:

$$
\begin{aligned}
& \frac{\mathrm{d}[R]}{\mathrm{d} t}=-1000 I_{0} \eta\left\{\frac{\left(1-10^{-A}\right)}{A}\right\}\left[\varepsilon_{R}[R]-\phi \frac{k_{i s c}}{k_{i s c}+k_{y}} A\right] \\
& \frac{\mathrm{d}[S]}{\mathrm{d} t}=-1000 I_{0} \eta\left\{\frac{\left(1-10^{-A}\right)}{A}\right\}\left[\varepsilon_{S}[S]-\phi \frac{k_{i s c}}{k_{i s c}+k_{y}} A\right]
\end{aligned}
$$

where $\phi$ is the recombination yield from ${ }^{1} \mathrm{X}$ to each enantiomer $\left(0<\phi=k_{\mathrm{c}} /\left(2 k_{\mathrm{c}}+k_{\mathrm{z}}\right)<0.5\right)$.

The second terms in equations (7) and (8) containing $k_{\mathrm{y}}, k_{\mathrm{isc}}$, and $\phi$ exhibit the magnetic field dependence if $k_{\text {isc }}$ is changed by applying a magnetic field. When $k_{\mathrm{y}}$ is zero, $k_{\text {isc }}$ in the second terms is canceled. Therefore, the presence of the escape process from ${ }^{3} \mathrm{X}$ is indispensable for observing the MFE. Besides, in the case of $\phi=0$, because the second terms vanish, large recombination yield from ${ }^{1} \mathrm{X}$ to each enantiomer is also necessary. In addition, it is the most important for obtaining large ee that the $g$ factor should be large enough, as mentioned earlier.

Computer simulations of the concentration changes in enantiomers were made by numerical integration of differential equations (7) and (8) by the Runge-Kutta method. ${ }^{39}$ Here, the values of $\varepsilon_{R}$ and $\varepsilon_{S}$ are fixed at 49 and $51 \mathrm{M}^{-1} \mathrm{~cm}^{-1}$, respectively, which corresponds to the $g$ factor of 0.04 . This value is comparable to typical $g$ values of chiral carbonyl compounds reported in the literature. ${ }^{5}$

The simulation results of ee as a function of time are shown in Fig. 1. Lines a-c indicate the increase in ee at three repre-

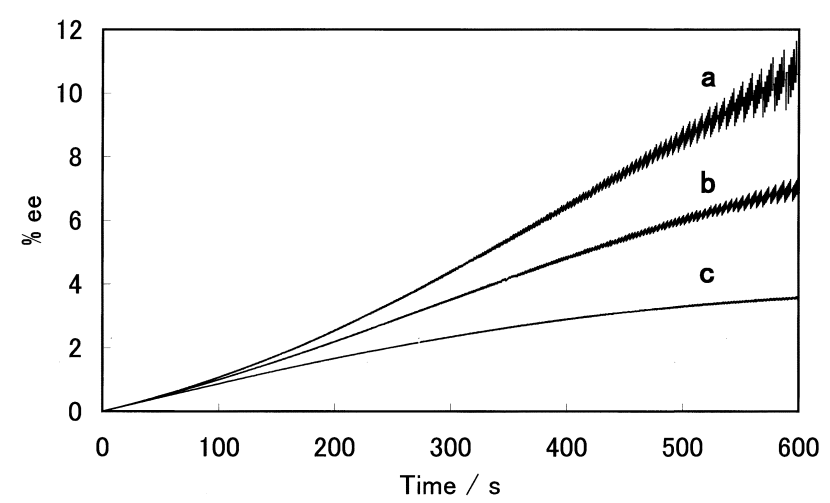

Fig. 1. Simulation results of the ee as a function of time. The rate constant $k_{\text {isc }}$ is treated as variable parameter; (a) $1 \times 10^{5} \mathrm{~s}^{-1}$, (b) $2 \times 10^{6} \mathrm{~s}^{-1}$, (c) $1 \times 10^{7} \mathrm{~s}^{-1} . I_{0}$ and $\eta$ are assumed to be $1 \times 10^{-7}$ einsteins $\cdot \mathrm{cm}^{-2} \mathrm{~s}^{-1}$ and unity, respectively. Values of $\varepsilon_{R}$ and $\varepsilon_{S}$ are fixed at 49 and $51 \mathrm{M}^{-1} \mathrm{~cm}^{-1}$, respectively. The rate constant $k_{\mathrm{y}}$ is set to be $1 \times 10^{7} \mathrm{~s}^{-1}$ and the recombination yield $\phi$ is fixed at 0.5 . Initial concentrations are $10 \mathrm{mM}$ for both enantiomers.

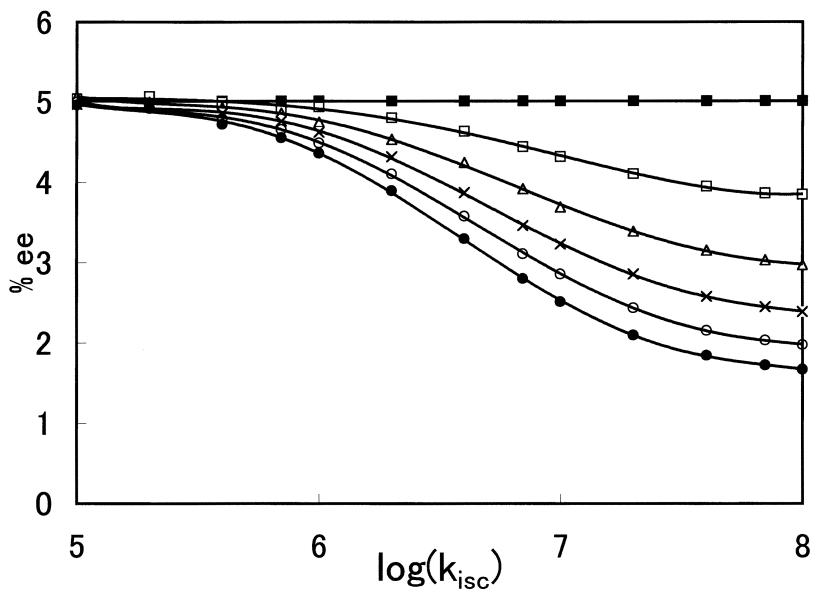

Fig. 2. The plots of ee versus logarithmic scales of $k_{\text {isc }}$ at 330 s. The rate constant $k_{\text {isc }}$ and the recombination yield $\phi$ are treated as variable parameters; the $\phi$ values; $(\mathbf{\square}) 0,(\square) 0.1$, $(\triangle) 0.2,(\times) 0.3,(\bigcirc) 0.4,(\bigcirc) 0.5$. The other parameters are set to the same values as in the case of Fig. 1.

sentative values of $k_{\text {isc }}$. Here, $k_{\mathrm{y}}$ is set to be $1 \times 10^{7} \mathrm{~s}^{-1}$ and $\phi$ is fixed at 0.5 , which means that all ${ }^{1} \mathrm{X}$ equally recombine to form each enantiomer. It is apparent that the values of ee depend on $k_{\text {isc }}$ and increase with decreasing $k_{\text {isc. }}$ Consequently, large MFE are expected in this kinetic model.

In order to obtain detailed conditions for the MFE, we evaluated ee as a function of $k_{\text {isc }}$ and $\phi$. Fig. 2 shows plots of the ee versus logarithmic values of $k_{\text {isc }}$ for each $\phi$ at $330 \mathrm{~s}$. Note that the variation in ee becomes larger with increasing $\phi$. This means that the large recombination yield is necessary for observing the MFE. In addition, it is noteworthy that the variation is significant in the range of $\log \left(k_{\text {isc }}\right)=6-7$, which is close to the escape rate $\left(k_{\mathrm{y}}=1 \times 10^{7} \mathrm{~s}^{-1}\right)$. Therefore, the ISC rate of a radical-pair or biradical should be nearly equal to the rate of an escape process. Moreover, the ISC rate should be changed over one order of magnitude upon applying a magnetic field, even if the recombination yield is sufficiently large.

\section{Experimental}

Sample Preparations: 2-Phenylcyclohexanone (2-PCH, $n=$ 6, Aldrich) and 2-phenylcycloheptanone (2-PCHep, $n=7$, Aldrich) were used after purification by column chromatopraphy. 2Phenylcyclooctanone (2-PCO, $n=8$ ) was prepared by the reaction of an enolate ion of cyclooctanone (Tokyo Kasei Kogyo) with diphenyliodonium trifluoromethanesulfonate (Tokyo Kasei Kogyo) in the presence of copper(I) cyanide (Katayama Kagaku), as reported by Ryan and Stang. ${ }^{40}$ Purification of synthesized 2-PCO was carried out by column chromatography $\left(\mathrm{SiO}_{2}, 6 \%\right.$ ethyl acetate in hexane) to yield a colorless oil $\left(6.4 \%\right.$ yield, ${ }^{1} \mathrm{H}-\mathrm{NMR}$ $\left(\mathrm{CDCl}_{3}\right) \delta 7.28(\mathrm{~m}, 5 \mathrm{H}), 3.79(\mathrm{dd}, J=12.0,4.2 \mathrm{~Hz}, 1 \mathrm{H}), 2.65-$ $1.20(\mathrm{~m}, 12 \mathrm{H})$ ). 2-Propanol of spectroscopy grade or HPLC grade (Nacalai Tesque) was used as received. Degassed solutions were made by several freeze-pump-thaw cycles. $\mathrm{O}_{2}$-saturated solutions were prepared by bubling pure $\mathrm{O}_{2}$ gas for $20 \mathrm{~min}$ before experiments.

Optical resolutions were conducted on a HPLC system (Shimadzu LC-6AD pump, a DGU-12A 2-line degasser, a CTO-10AS column oven, and a SPD-10A UV-vis detector) equipped with a normal-phase chiral column (Daicel Chiralcel OF for 2-PCH and 
Daicel Chiralpak AS for 2-PCHep and 2-PCO, $4.6 \mathrm{~mm}$ i.d. $\times 250$ $\mathrm{mm})$. 2-propanol/hexane $(1: 9)$ at a flow rate of $0.5 \mathrm{ml} / \mathrm{min}$ was used as an eluent. A chromatogram was monitored at $265 \mathrm{~nm}$.

Spectroscopic Measurements: UV absorption spectra were measured on a Hitachi U-3210 spectrophotometer. CD spectra were obtained on a JASCO J-725S spectropolarimeter. Optical rotations were measured using a Horiba SEPA-300 polarimeter with a $10 \mathrm{~cm}$ path length.

Transient Absorption Measurements: The experimental apparatus for transient-absorption measurements were similar to those reported previously. ${ }^{12-16}$ In the case of a magnetic field strength below $1 \mathrm{~T},{ }^{12,15,16}$ an excimer laser (Lambda Physik CompEX 100, XeCl at $308 \mathrm{~nm}$, fwhm ca. $15 \mathrm{~ns}, 100 \mathrm{~mJ} /$ pulse) was used as the exciting light source and a xenon flash lamp (EG\&G FXQ-853) was used as the monitoring light source. Sample solutions were placed between the pole pieces of an electromagnet (Tokin SEE-9G). The magnetic field strength was controlled by a current-regurated power supply. In the case of a high field strength, a pulse magnet-laser flash photolysis apparatus was used. $^{13,14}$ A pulsed magnetic field (1-8 T, 2ms) was generated by supplying an intense pulsed current from a condenser bank (Nichikon, $5 \mathrm{kV}, 50 \mathrm{~kJ}$ ) to a home-made solenoid $(9.7 \mathrm{mH}$ and 0.7 $\Omega$ ). The exciting and monitoring light sources were the fourth harmonics (266 nm, fwhm 4-6 ns, $10 \mathrm{~mJ} /$ pulse) of an Nd: YAG laser (Spectra Physics, GCR-11-1) and a Xe arc lamp (Ushio, 150 $\mathrm{W})$, respectively. The laser pulse and the monitoring light were introduced coaxially to a sample cell, and then light was passed through the sample solution and then detected using optical fibers. All of the measurements were carried out at $20-23{ }^{\circ} \mathrm{C}$.

Photoproducts Analyses: $\mathrm{O}_{2}$-saturated 2-PCAs solutions (2 $\mathrm{mM})$ in a cylindrical quartz cell $(\phi 2.5 \mathrm{~cm} \times 5 \mathrm{~cm}$ path length) were irradiated with UV light from a 500-W high-pressure mercury lamp (Ushio) equipped with a glass filter (Toshiba UV-29) for $20 \mathrm{~min}$ for 2-PCH and 2-PCHep and $10 \mathrm{~min}$ for 2-PCO. After irradiation, the solutions were concentrated to about $1 / 10$ volume. Photoproduct analyses were carried out on a multi-channel UVdetected HPLC system (a Waters 510 pump, a Shimazdu CTO10AS column oven, a Otsuka Electronics MCPD 3600 multichannel photodetector, 2-propanol/hexane (1:9) eluent with a flow rate of $0.5 \mathrm{~mL} / \mathrm{min}$ ) with a chiral column (Daicel Chiralcel OF) and a GC/MS instrument (HP-5890GC/JEOL JMS AX5) equipped with a chiral capillary column (Supelco $\gamma$-DEX 225, 30 $\mathrm{m} \times 0.25 \mathrm{~mm}$ i. d.). Helium carrier gas was used with a linear velocity of $30 \mathrm{~cm} / \mathrm{s}$. A sample solution $(3 \mu \mathrm{L})$ was injected with a split ratio of $20: 1$. The injector temperature was $250{ }^{\circ} \mathrm{C}$, and the column temperature program, which began at $100{ }^{\circ} \mathrm{C}$, was held for 2 min and ramped at $3{ }^{\circ} \mathrm{C} / \mathrm{min}$ to $190{ }^{\circ} \mathrm{C}$. The mass spectrometer was set to scan mass units of 20-350 for electron impact ionization with a source temperature of $230{ }^{\circ} \mathrm{C}$. Chromatograms were obtained at a rate of $36 \mathrm{scan} / \mathrm{min}$.

Photolyses with Linearly and Circularly Polarized Light: The XeCl pulsed laser beam (Lambda Physik CompEX 100; frequency, $10 \mathrm{~Hz}$; fwhm, ca. $15 \mathrm{~ns} ; 308 \mathrm{~nm}, 100 \mathrm{~mJ} /$ pulse) was first converted into linearly polarized light (LPL) by passing through a circular iris $(\phi=10 \mathrm{~mm})$ and a polarizer (Sigma Koki, glan laser prism, $10 \times 10 \mathrm{~mm}, 21 \mathrm{~mm}$ thick). LPL, left- and right-CPL were obtained by passing the incident LPL through a phase-adjusted Babinet-Soleil compensator (Oyodenko, $\phi=8 \mathrm{~mm}$ ) oriented with angles of $0^{\circ}$ for LPL, $-45^{\circ}$ for left-CPL, and $+45^{\circ}$ for right-CPL with respect to the plane of polarization of the incident LPL. The average output of the final polarized light was ca. $2 \mathrm{~mJ} / \mathrm{cm}^{2} \cdot$ pulse.
Air-saturated sample solutions $(4 \mathrm{mM})$ in a cylindrical quartz cell ( $\phi=8 \mathrm{~mm}, 10 \mathrm{~mm}$ path length) were placed between the pole pieces of an electromagnet (Tokin SEE-9G). The enantiomeric excess and the extent of 2-PCAs photodecomposition were determined on a gas chromatograph (Shimadzu GC-14A) equipped with a chiral capillary column (Supelco $\gamma$-DEX 225, $30 \mathrm{~m} \times 0.25$ $\mathrm{mm}$ i. d.). Helium carrier gas was used with a linear velocity of 30 $\mathrm{cm} / \mathrm{s}$. A sample solution $(1 \mu \mathrm{L})$ was injected using a splitless mode. The injector temperature was $250^{\circ} \mathrm{C}$ and the FID temperature was $300{ }^{\circ} \mathrm{C}$. The column temperature program began at 120 ${ }^{\circ} \mathrm{C}$, was held for $2 \mathrm{~min}$ and then ramped at $2{ }^{\circ} \mathrm{C} / \mathrm{min}$ to $180^{\circ} \mathrm{C}$. 1 , 10-Decanediol or 1, 12-dodecanediol was used as an internal standard.

\section{Results}

1. Optical and Chiroptical Properties: The three 2PCAs were completely resolved using chiral HPLC methods. The absolute configuration of the resolved 2-PCH was determined by comparing the sign of the optical rotation of our sample solutions with reference data. ${ }^{41,42}$ As for 2-PCHep and 2-PCO, the sign of the optical rotation has not yet been correlated with the absolute stereochemical configuration.

The absorption, CD, and anisotropy $g$ factor spectra of 2PCAs are shown in Fig. 3. The absorption spectra $(\mathrm{a}-\mathrm{c})$ of these compounds mainly consist of a broad $n \pi^{*}$ band of carbonyl group in $280-330 \mathrm{~nm}$ region and a vibronic $\pi \pi^{*}$ band of phenyl group $\left({ }^{1} \mathrm{~A}_{1 \mathrm{~g}} \rightarrow{ }^{1} \mathrm{~B}_{2 \mathrm{u}}\right)$ around $260 \mathrm{~nm}$. Intensity of the forbidden $n \pi^{*}$ transition increases with size of the cycloalkanone rings. This is due to an amplification of the $n \pi{ }^{*}$ transition probability by an interaction with the $\pi \pi^{*}$ transition of phenyl group caused by a ring distortion of 2-PCHep and 2$\mathrm{PCO}^{43}$ Absorption bands $(<250 \mathrm{~nm})$ which appeared for 2PCHep and 2-PCO can probably be ascribed to the second $\pi \pi^{*}$ transition of phenyl group $\left({ }^{1} \mathrm{~A}_{1 \mathrm{~g}} \rightarrow{ }^{1} \mathrm{~B}_{1 \mathrm{u}}\right)$, which may be coupled with the $n \pi^{*}$ transition of the carbonyl group because CD signals are observed in this region. ${ }^{43}$

Since the $n \pi^{*}$ band of the carbonyl groups mainly contributes to the CD spectra of 2-PCAs (see spectra d-f in Fig. 3), the anisotropy $g$ factor calculated from $\mathrm{CD}$ and absorption spectra indicates the maximum values around $300 \mathrm{~nm}$. This is the reason why we chose the excitation wavelength to be 308 $\mathrm{nm}$ for all 2-PCAs. The molar extinction coefficients $(\varepsilon), \mathrm{CD}$ intensities $(\Delta \varepsilon)$, and calculated $g$ factors $(\Delta \varepsilon / \varepsilon)$ at $308 \mathrm{~nm}$ are summarized in Table 1. The $g$ value of 2-PCH is the largest because of the smallest $n \pi{ }^{*}$ transition intensity. Therefore, the ee value of 2-PCH induced by CPL irradiation is expected to be the largest of the three 2-PCAs.

2. Photoproducts in the Presence of Molecular Oxygen: The photolysis mechanism of 2-PCAs is depicted in Scheme 1, in which the Norrish type I photocleavage of the excited triplet 2-PCAs yields the acyl-benzyl biradical. Four types of product formation processes have been identified as (1) recombination to the starting 2-PCAs, ${ }^{26-28}$ (2) intramolecular disproportionation (hydrogen abstraction) to phenylalkenals, ${ }^{32,34-38}$ (3) para-coupling to cyclophanes for large ring 2-PCAs $(n=10$ $15), 32,36,37$ and (4) bimolecular radical scavenging processes (escape process) with $\mathrm{CCl}_{4}, \mathrm{CBrCl}_{3}$ or $\mathrm{O}_{2} \cdot{ }^{27,28}$ In these processes, the escape process from the triplet acyl-benzyl biradical is indispensable for observing the MFE, as mentioned ear- 

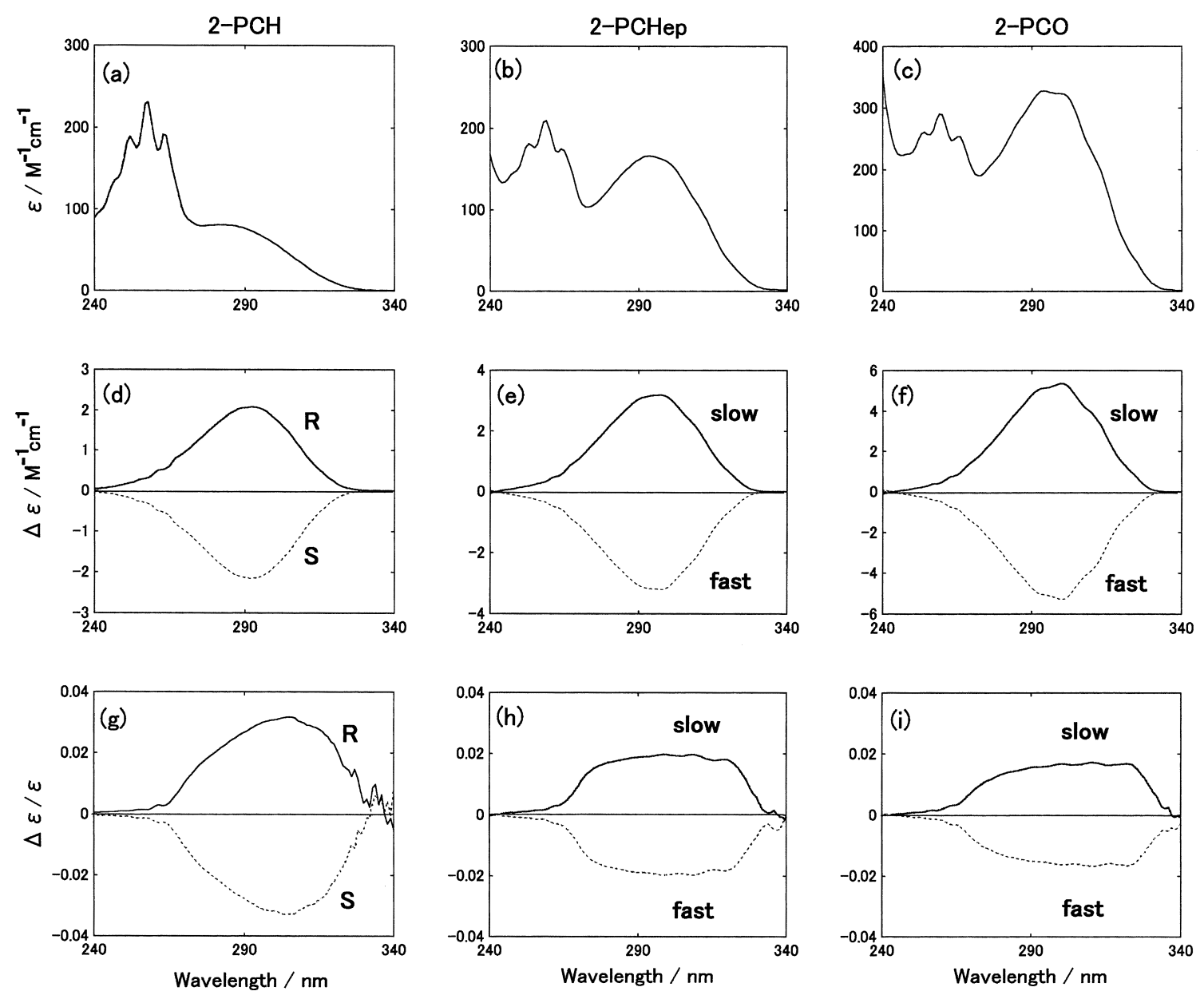

Fig. 3. Absorption (a)-(c), CD (d)-(f), and $g$ factor (g)-(i) spectra of 2-PCAs in 2-propanol in near UV region (240-340 nm). The faster effluent enantiomers of 2-PCHep and 2-PCO on a chiral-capirally GC are indicated by broken lines (fast) in CD and $g$ factor spectra [(e), (f), (h) and (i)]. The slower ones for 2-PCHep and 2-PCO are also indicated by solid lines (slow) in the spectra [(e), (f), (h) and (i)].

Table 1. Molar Extinction Coefficients $(\varepsilon)$, Circular Dichroism $(\Delta \varepsilon)$, and Anisotropy $g$ Factors of 2-PCAs at $308 \mathrm{~nm}$

\begin{tabular}{lccc}
\hline 2-PCAs & $\varepsilon / \mathrm{M}^{-1} \mathrm{~cm}^{-1}$ & $\Delta \varepsilon / \mathrm{M}^{-1} \mathrm{~cm}^{-1}$ & $g$ factor \\
\hline 2-PCH & 36.6 & 1.1 & 0.030 \\
2-PCHep & 117.2 & 2.3 & 0.020 \\
2-PCO & 249.1 & 4.2 & 0.017 \\
\hline
\end{tabular}

lier. Therefore, we are especially interested in the $\mathrm{O}_{2}$ concentration dependence on photoproduct formations which can be investigated by a GC/MS analysis.

Figure 4 shows GC/MS total ion chromatograms of the photoproducts in $\mathrm{O}_{2}$-saturated solutions. The retention times on GC and the mass-to-charge ratio $(\mathrm{m} / \mathrm{z})$ of molecular and fragment ions of the photoproducts and starting 2-PCAs are summarized in Table 2. In the chromatogram of 2-PCH, two peaks $((R)$ and $(S))$ are assigned to the starting $R$ and $S$ enantiomers of 2-PCH, indicating that a pair of enantiomer is well-separated on this chiral capillary GC. Peaks (a) and (b) are attributed to 3-butenoic acid and acetophenone by a comparison with an authentic sample. Peak (c) has a mass spectrum with a molecular ion at $m / z 174$, which suggests that this component is due to an isomer of the starting material. Additionally, absorption spectrum recorded for peak (c) on UV-detected HPLC indicates that this component has a $\beta$-alkylstyrene chromophore. Therefore, peak (c) is assigned to 6-phenyl-5-hexenal ( $\mathrm{Ph}$ $\left.\mathrm{CH}=\mathrm{CH}\left(\mathrm{CH}_{2}\right)_{3} \mathrm{CHO}\right)$, which is mainly obtained in a degassed solution. ${ }^{34-38}$ The peaks in the chromatograms of 2-PCHep and 2-PCO were also identified in the same manner for 2-PCH. Pairs of enantiomers for 2-PCHep and 2-PCO are also separated well. Other trace peaks in the three chromatograms have not been identified. No peaks assigned to phenylalkanoic acids were detected in this work, whereas Doubleday reported CIDNP signals due to these species, which may derived from a ketene intermediate. ${ }^{26}$ The ketene was considered to be formed through disproportionation (hydrogen abstraction from the methylene group next to the acyl moiety by the phenylalkyl radical).

Acetophenone and alkenoic acids were not detected in degassed solutions at all. It is further worth noting that the peak intensities of phenylalkenals (c) decreased with increasing $\mathrm{O}_{2}$ concentration, in contrast to increasing those of acetophenone (b) and alkenoic acids (a). This opposite dependence on the $\mathrm{O}_{2}$ concentration can be explained by the reaction of the triplet biradical with molecular oxygen dissolved in solutions, and thereby the product yield of phenylalkenals is decreased with 

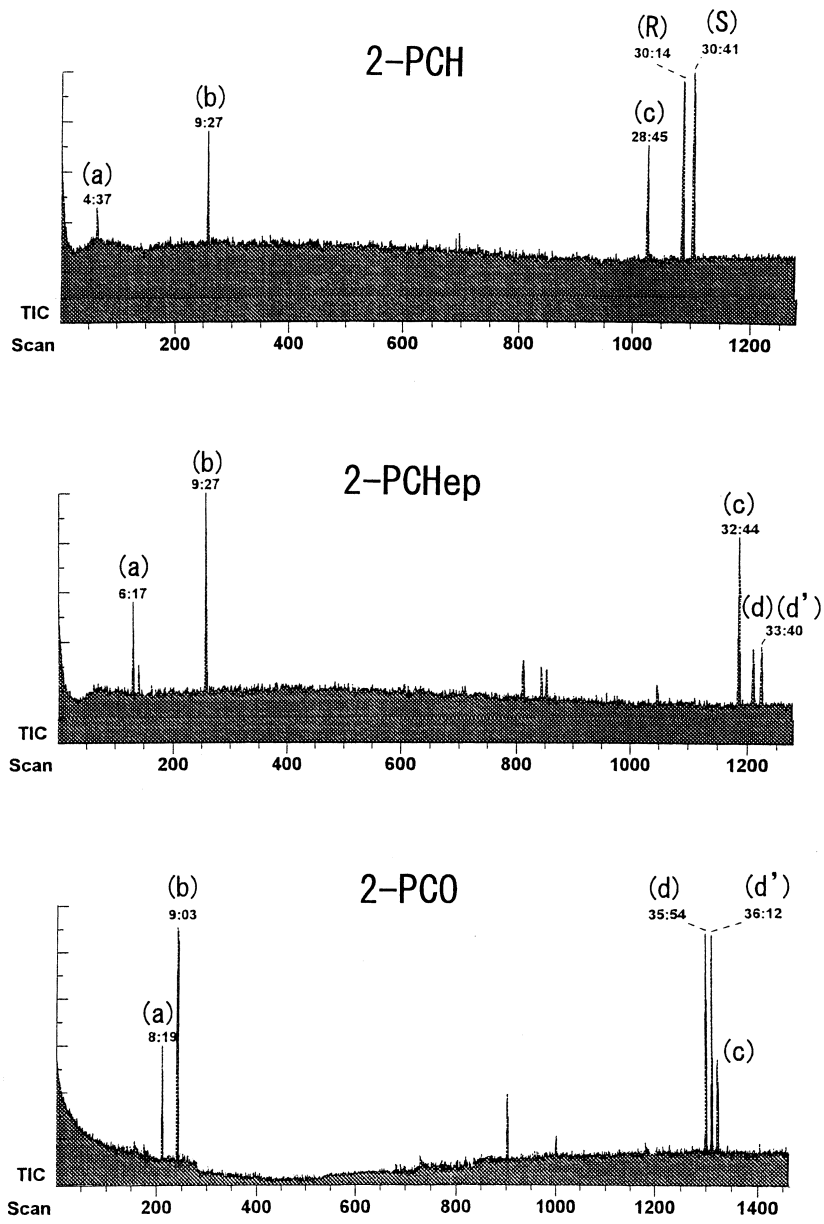

Fig. 4. Total ion chromatograms of photoproducts and starting 2-PCAs in $\mathrm{O}_{2}$ saturated 2-propanol solutions after UV irradiations $(>290 \mathrm{~nm})$ of $20 \mathrm{~min}$ for 2-PCH and 2PCHep and $10 \mathrm{~min}$ for 2-PCO. See Table 2 for assignment of peaks. Extents of sample decompositions were $54 \%$ for 2-PCH, $86 \%$ for 2-PCHep and $80 \%$ for 2-PCO. The chromatograms were obtained at the rate of $36 \mathrm{scan} / \mathrm{min}$. The detailed analytical conditions are described in the text.

$\mathrm{O}_{2}$ concentration.

3. Biradical Lifetimes: In order to confirm the reaction between the triplet biradical and $\mathrm{O}_{2}$, we measured transient absorption signals of the benzyl radical moiety $(320 \mathrm{~nm})$ on triplet acyl-benzyl biradicals generated upon $308 \mathrm{~nm}$ laser flash photolysis. Figure 5 shows transient absorption decay curves of 2-PCH in degassed, air- and $\mathrm{O}_{2}$-saturated solutions. Obviously the transient signal of decay component of the biradical is quenched in the presence of molecular oxygen. The plateau signal appeared for the air- and $\mathrm{O}_{2}$-satureated solutions are presumably due to permanent photoproducts because its signal remains on a time scale longer than millisecond. Similar results are obtained for 2-PCHep and 2-PCO.

These transient absorption singals were well-fitted with $\operatorname{Abs}(t)=A+B \exp (-t / \tau)$, where $\operatorname{Abs}(\mathrm{t})$ is the transient absorption intensity, $A$ and $B$ are time-independent constants and $\tau$ is the triplet biradical lifetime. The obtained lifetimes for degassed $\left(\tau_{\mathrm{deg}}\right)$ and air-saturated $\left(\tau_{\mathrm{air}}\right)$ solutions are listed in Table 3. The $\tau_{\mathrm{deg}}$ values for all 2-PCAs are in agreement with those
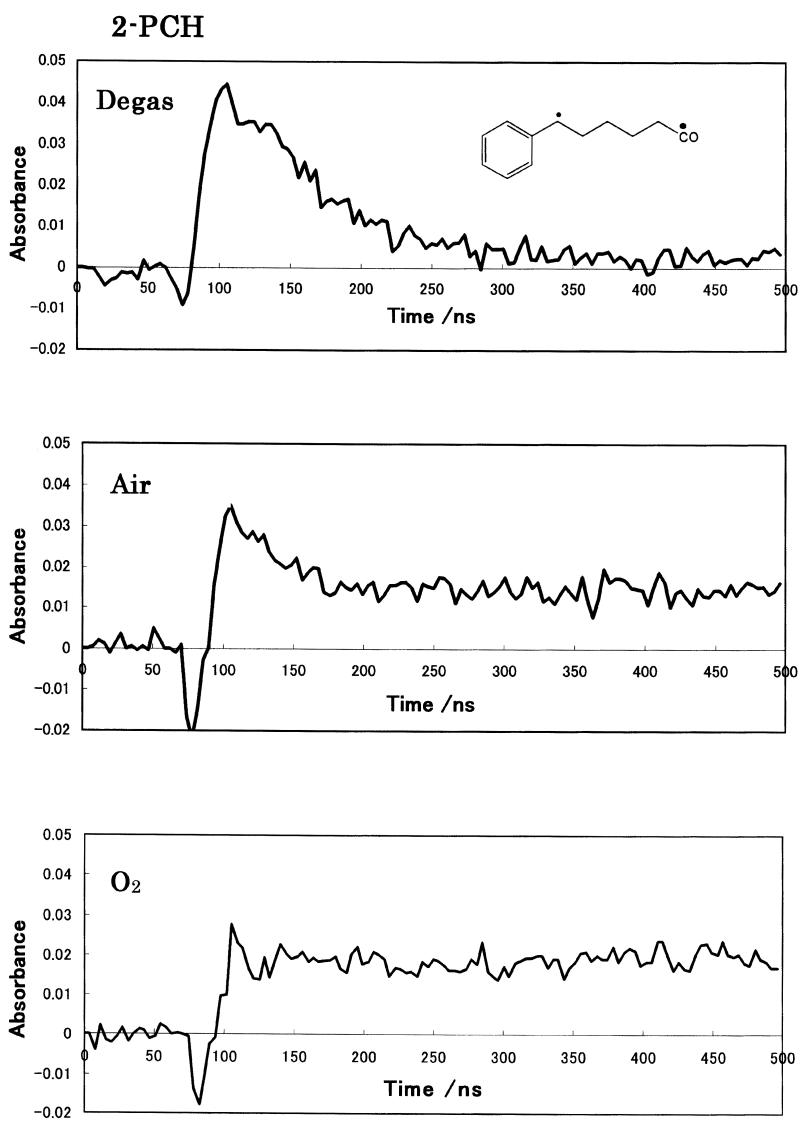

Fig. 5. Transient absorption decay profiles of 2-PCH monitored at $320 \mathrm{~nm}$ in degassed (upper), air saturated (middle), and $\mathrm{O}_{2}$ saturated (lower) solutions. The excitation light source was a $\mathrm{XeCl}$ eximer laser $(100 \mathrm{~mJ} /$ pulse, fwhm ca. $15 \mathrm{~ns}, 308 \mathrm{~nm})$.

in methanol and cyclohexanol reported by Doubleday and Turro et al. ${ }^{30,32}$ From previous studies, ${ }^{30-33}$ ISC is regarded as being the only deactivation process of the triplet biradicals in degassed solutions. In addition, reverse ISC from singlet to triplet biradical is negligible because the rates of recombination and disproportionation from the singlet biradical are much larger than that of the ISC. Therefore, the reciprocal of $\tau_{\mathrm{deg}}$ corresponds to the ISC rate constant $\left(k_{\text {isc }}\right)$ of the triplet biradical. In air-saturated solutions, however, because the escape reaction with $\mathrm{O}_{2}$ is the second deactivation process of the triplet biradical, the reciprocal of the lifetime $\left(\tau_{\text {air }}\right)$ is expressed as $1 / \tau_{\text {air }}=k_{\text {isc }}+k_{\text {esc }}\left[\mathrm{O}_{2}\right]$, where $k_{\text {esc }}$ is a bimolecular rate constant and $\left[\mathrm{O}_{2}\right]$ is the $\mathrm{O}_{2}$ concentration dissolved in the solution. Assuming that $\left[\mathrm{O}_{2}\right]$ is $2.2 \mathrm{mM}$ in 2-propanol at $20{ }^{\circ} \mathrm{C},{ }^{44}$ the values of $k_{\text {esc }}$ can be estimated. These kinetic parameters $\left(k_{\mathrm{isc}}, k_{\mathrm{esc}}\left[\mathrm{O}_{2}\right]\right.$ and $\left.k_{\text {esc }}\right)$ are summarized in Table 3 . The values of $k_{\text {esc }}\left[\mathrm{O}_{2}\right]$ in air-saturated solutions are nearly equal to those of $k_{\text {isc }}$ for all 2PCAs. This fact is very important for observing MFE in asymmetric photolysis with CPL, as mentioned earlier. The values of $k_{\text {esc }}$ are in the range of $10^{9}-10^{10} \mathrm{M}^{-1} \mathrm{~s}^{-1}$, indicating that the triplet biradical undergoes a diffusion-controlled reaction with $\mathrm{O}_{2}$.

The lifetimes of the triplet biradicals at a field strength of $0.96 \mathrm{~T}\left(\tau_{\mathrm{MG}}\right)$ are listed in Table 3 . It can be seen that there is no differences between the values of $\tau_{\mathrm{deg}}$ (in the Earth magnet- 
Table 2. Retention Time on Gas-Chromatography and Mass to Charge Ratio $(\mathrm{m} / \mathrm{z})$ of Molecular $\left(\mathbf{M}^{+}\right)$and Fragment Ions on Mass Spectra of Photoproducts and 2-PCAs

\begin{tabular}{|c|c|c|c|c|}
\hline 2-PCAs & Peak & $\begin{array}{l}\text { Retention time } \\
/ \min \left({ }^{\prime}\right) \mathrm{s}\left({ }^{\prime \prime}\right)\end{array}$ & $\begin{array}{l}m / z \text { of molecular }\left(\mathrm{M}^{+}\right) \\
\text {and fragment ions }\end{array}$ & 2-PCAs and photoproducts \\
\hline \multirow{5}{*}{ 2-PCH } & $\mathrm{a}$ & $4^{\prime} 37^{\prime \prime}$ & $86\left(\mathrm{M}^{+}\right)$ & $\begin{array}{l}\text { 3-butenoic acid } \\
\left(\mathrm{CH}_{2}=\mathrm{CHCH}_{2} \mathrm{CO}_{2} \mathrm{H}\right)\end{array}$ \\
\hline & $\mathrm{b}$ & $9^{\prime} 27^{\prime \prime}$ & $120\left(\mathrm{M}^{+}\right), 105\left(\mathrm{M}^{+}-\mathrm{CH}_{3}\right)$ & acetophenone \\
\hline & $\mathrm{c}$ & $28^{\prime} 45^{\prime \prime}$ & $174\left(\mathrm{M}^{+}\right), 130\left(\mathrm{M}^{+}-\mathrm{CH}_{3} \mathrm{CHO}\right)$ & $\begin{array}{l}\text { 6-phenyl-5-hexenal } \\
\left(\mathrm{PhCH}=\mathrm{CH}\left(\mathrm{CH}_{2}\right)_{3} \mathrm{CHO}\right)\end{array}$ \\
\hline & $R$ & $30^{\prime} 14^{\prime \prime}$ & $174\left(\mathrm{M}^{+}\right)$ & 2-PCH, $R$ enantiomer \\
\hline & $S$ & $30^{\prime} 41^{\prime \prime}$ & $174\left(\mathrm{M}^{+}\right)$ & 2-PCH, $S$ enantiomer \\
\hline \multirow{5}{*}{ 2-PCHep } & $\begin{array}{ll}- \\
\mathrm{a}\end{array}$ & $6^{\prime} 17^{\prime \prime}$ & $100\left(\mathrm{M}^{+}\right)$ & $\begin{array}{l}\text { 4-pentenoic acid } \\
\left(\mathrm{CH}_{2}=\mathrm{CH}\left(\mathrm{CH}_{2}\right)_{2} \mathrm{CO}_{2} \mathrm{H}\right)\end{array}$ \\
\hline & $\mathrm{b}$ & $9^{\prime} 27^{\prime \prime}$ & $120\left(\mathrm{M}^{+}\right), 105\left(\mathrm{M}^{+}-\mathrm{CH}_{3}\right)$ & acetophenone \\
\hline & $\mathrm{c}$ & $32^{\prime} 44^{\prime \prime}$ & $\begin{array}{l}188\left(\mathrm{M}^{+}\right), 170\left(\mathrm{M}^{+}-\mathrm{H}_{2} \mathrm{O}\right) \\
144\left(\mathrm{M}^{+}-\mathrm{CH}_{3} \mathrm{CHO}\right)\end{array}$ & $\begin{array}{l}\text { 7-phenyl-6-hexenal } \\
\left(\mathrm{PhCH}=\mathrm{CH}\left(\mathrm{CH}_{2}\right)_{4} \mathrm{CHO}\right)\end{array}$ \\
\hline & $\mathrm{d}$ & $33^{\prime} 20^{\prime \prime}$ & $188\left(\mathrm{M}^{+}\right)$ & 2-PCHep \\
\hline & $\mathrm{d}^{\prime}$ & $33^{\prime} 40^{\prime \prime}$ & $188\left(\mathrm{M}^{+}\right)$ & 2-PCHep \\
\hline \multirow{6}{*}{ 2-PCO } & - - & $-\cdots$ & - & $\ldots-\ldots$ \\
\hline & a & $8^{\prime} 19^{\prime \prime}$ & $114\left(\mathrm{M}^{+}\right)$ & $\begin{array}{l}\text { 5-hexenoic acid } \\
\left(\mathrm{CH}_{2}=\mathrm{CH}\left(\mathrm{CH}_{2}\right)_{3} \mathrm{CO}_{2} \mathrm{H}\right)\end{array}$ \\
\hline & $\mathrm{b}$ & $9^{\prime} 03^{\prime \prime \dagger}$ & $120\left(\mathrm{M}^{+}\right), 105\left(\mathrm{M}^{+}-\mathrm{CH}_{3}\right)$ & acetophenone \\
\hline & $\mathrm{d}$ & $35^{\prime} 54^{\prime \prime}$ & $202\left(\mathrm{M}^{+}\right)$ & 2-PCO \\
\hline & $\mathrm{d}^{\prime}$ & $36^{\prime} 12^{\prime \prime}$ & $202\left(\mathrm{M}^{+}\right)$ & 2-PCO \\
\hline & $\mathrm{c}$ & $36^{\prime} 30^{\prime \prime}$ & $\begin{array}{l}202\left(\mathrm{M}^{+}\right), 184\left(\mathrm{M}^{+}-\mathrm{H}_{2} \mathrm{O}\right) \\
156\left(\mathrm{M}^{+}-\mathrm{CH}_{3} \mathrm{CHO}\right)\end{array}$ & $\begin{array}{l}\text { 7-phenyl-6-hexenal } \\
\left(\mathrm{PhCH}=\mathrm{CH}\left(\mathrm{CH}_{2}\right)_{5} \mathrm{CHO}\right)\end{array}$ \\
\hline
\end{tabular}

$\dagger$ The slight difference of retention time of acetophenone between 2-PCO and 2-PCH (2-PCHep) is due to shortened length of the capillary column.

Table 3. Lifetimes of Triplet Acyl-Benzyl Biradicals and Other Kinetic Parameters

\begin{tabular}{lcccccc}
\hline & $\tau_{\text {air }} / \mathrm{ns}$ & $\tau_{\text {deg }} / \mathrm{ns}$ & $\tau_{\mathrm{MF}}^{\mathrm{a}} / \mathrm{ns}$ & $k_{\mathrm{isc}}^{\mathrm{b})} / 10^{7} \mathrm{~s}^{-1}$ & $k_{\mathrm{esc}}\left[\mathrm{O}_{2}\right]^{\mathrm{c})} / 10^{7} \mathrm{~s}^{-1}$ & $k_{\mathrm{esc}} / 10^{9} \mathrm{M}^{-1} \mathrm{~s}^{-1}$ \\
\hline 2-PCH & $35 \pm 2$ & $65 \pm 3$ & $67 \pm 2$ & 1.5 & 1.3 & 6.2 \\
2-PCHep & $49 \pm 2$ & $85 \pm 3$ & $86 \pm 3$ & 1.2 & 0.8 & 3.8 \\
2-PCO & $37 \pm 4$ & $92 \pm 4$ & $90 \pm 2$ & 1.1 & 1.6 & 7.3 \\
\hline
\end{tabular}

a) Magnetic field strength is $0.96 \mathrm{~T}$.

b) $k_{\text {isc }}=1 / \tau_{\text {deg }}$.

c) $k_{\text {esc }}\left[\mathrm{O}_{2}\right]=1 / \tau_{\text {air }}-k_{\text {isc }}$, where $\left[\mathrm{O}_{2}\right]$ is assumed to be $2.2 \mathrm{mM}$ in 2-propanol at $20^{\circ} \mathrm{C}^{44}$

ic field) and $\tau_{\mathrm{MG}}$ within the experimental error. Although we examined the triplet biradical lifetime in a higher field region (1-8 T) for 2-PCH and 2-PCHep, the lifetimes remain unchanged. This result clearly indicates that the ISC rates of these biradicals are not affected by a static magnetic field below $8 \mathrm{~T}$.

4. Time Evolution of Enantiomer Concentrations: According to the results of our kinetic simulation, large recombination yield of the singlet biradical to each enantiomer is found to be one of the necessary conditions to obtain large MFE. This recombination reaction has already been demonstrated by Doubleday's CIDNP study. ${ }^{26}$ In addition, the rate constants for recombination were estimated theoretically by de Kanter and Kaptein $^{45}$ based on Doubleday's CIDNP data. ${ }^{26}$ In our approach, the recombination yield was evaluated by the following procedures: (A) The time evolution of the enantiomer concentration of 2-PCAs, starting with the one optically pure enantiomer, was monitored using chiral capillary GC. (B) The observed time dependence was simulated by integrating differential Eqs. 7 and 8 based on the Runge-Kutta method, where the recombination yield was treated as a fitting parameter.

Figure 6 shows the time evolution of the enantiomer concentrations of 2-PCAs with LPL irradiation. It is clear that as the one enantiomer concentration decreases, the other enantiomer concentration increases to reach the maximum value, and then decrease. Finally, both enantiomer concentrations coincide asymptotically. This result clearly indicates that the racemization of 2-PCAs occurs via photoproduction and recombination of the biradicals.

The solid curves in Fig. 6 are fitting results, in which $k_{\mathrm{y}}$ (= $\left.k_{\text {esc }}\left[\mathrm{O}_{2}\right]\right), k_{\text {isc }}, \varepsilon_{R}$, and $\varepsilon_{S}$ in the Eqs. 7 and 8 are fixed to the values obtained from our absorption, $\mathrm{CD}$, and transient absorption measurements, as listed in Tables 1 and 3 , whereas $I_{0} \eta$ and $\phi$ (recombination yield) are treated as variable parameters. From the fitting procedures, sum of the recombination yield to each enantiomer $(2 \phi)$ were determined as listed in Table 4 . $2 \phi$ was 

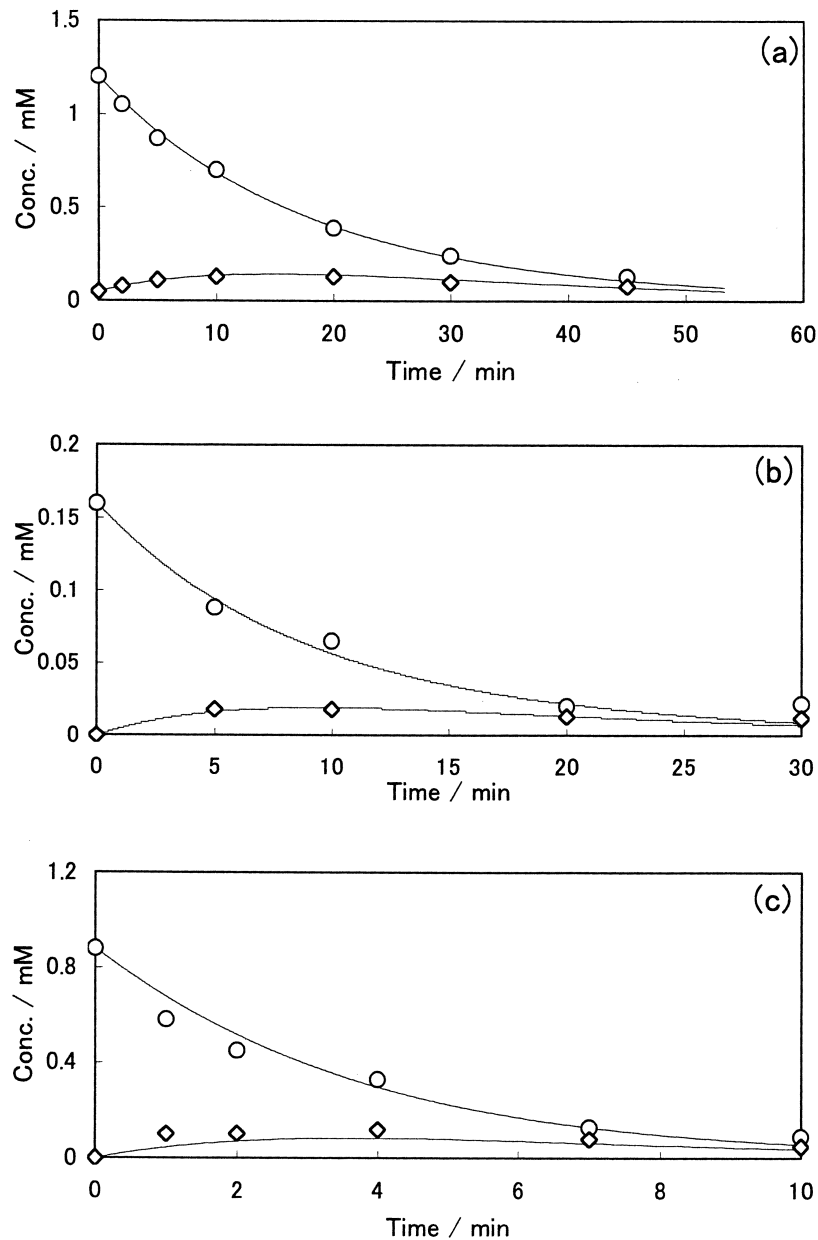

Fig. 6. Time evolution of 2-PCAs enantiomer concentration started from optically pure enantiomer upon pulsed linearly polarized light irradiation $(308 \mathrm{~nm}, 2 \mathrm{~mJ} /$ pulse, fwhm ca. $15 \mathrm{~ns})$ : (a) $2-\mathrm{PCH}(\bigcirc: R$ enantiomer, $\diamond: S$ enantiomer), (b) 2-PCHep and (c) 2-PCO. Curve fittings were made by numerical integration of equation (7) and (8) with parameters as follows; (a) $I_{0}: 5 \times 10^{-8}$ einsteins $\cdot \mathrm{cm}^{-2} \mathrm{~s}^{-1}, \eta: 0.30$, $\varepsilon_{R}=\varepsilon_{S}: 36.6 \mathrm{M}^{-1} \mathrm{~cm}^{-1}, k_{\mathrm{isc}}: 1.5 \times 10^{7} \mathrm{~s}^{-1}, k_{\mathrm{y}}: 1.3 \times 10^{7} \mathrm{~s}^{-1}$, $\phi: 0.4$, (b) $I_{0}: 5 \times 10^{-8}$ einsteins $\cdot \mathrm{cm}^{-2} \mathrm{~s}^{-1}, \eta: 0.18, \varepsilon_{R}=\varepsilon_{S}:$ $117.2 \mathrm{M}^{-1} \mathrm{~cm}^{-1}, k_{\mathrm{isc}}: 1.2 \times 10^{7} \mathrm{~s}^{-1}, k_{\mathrm{y}}: 0.8 \times 10^{7} \mathrm{~s}^{-1}, \phi:$ 0.4 , (c) $I_{0}: 5 \times 10^{-8}$ einsteins $\cdot \mathrm{cm}^{-2} \mathrm{~s}^{-1}, \eta: 0.24, \varepsilon_{R}=\varepsilon_{S}$ : $249.1 \mathrm{M}^{-1} \mathrm{~cm}^{-1}, k_{\text {isc }}: 1.1 \times 10^{7} \mathrm{~s}^{-1}, k_{\mathrm{y}}: 1.6 \times 10^{7} \mathrm{~s}^{-1}, \phi: 0.5$.

Table 4. Comparison of the Recombination Yield of AcylBenzyl Biradical $(2 \phi)$ Obtained in Our Experiments and Reference Values $(\Phi)$ Reported by de Kanter and Kaptein $^{45}$

\begin{tabular}{lcc}
\hline & $2 \phi^{\dagger}$ & $\Phi^{\ddagger}$ \\
\hline 2-PCH & 0.8 & 0.72 \\
2-PCHep & 0.8 & 0.80 \\
2-PCO & 1.0 & 0.91 \\
\hline
\end{tabular}

$\dagger$ A sum of recombination yield to each enantiomer. $\$$ Calculated by the following equation; $\Phi=k_{\mathrm{cyc}} /\left(k_{\mathrm{cyc}}+k_{\mathrm{ald}}\right.$ $+k_{\mathrm{ket}}$ ) where $k_{\mathrm{cyc}}, k_{\mathrm{ald}}$ and $k_{\mathrm{ket}}$ are rate constants for the formation of parent 2-PCAs, alkenals and ketenes, respectively, which are described in Table 1 in Ref. 45. estimated to be more than 0.8 for all 2-PCAs, and thus obtained large values. These values agree approximately with those obtained from simulations for the CINDP data ${ }^{26}$ by de Kanter and Kaptein, ${ }^{45}$ as listed in Table 4.

5. Asymmetric Photolysis in Air Saturated Solution: After racemic 2-PCAs were photolyzed with CPL in both the presence and absence of a magnetic field, the photoinduced ee were determined by a chiral capillary GC. The values of ee after ca. $90 \%$ sample decomposition are summarized in Table 5. For 2-PCH, $R$ enantiomer enrichment is achieved to about $3 \%$ ee by right-CPL irradiation and $S$ enantiomer vice versa. This value is consistent with our prediction based on a kinetic simulation, as discussed later. 2-PCH has the largest ee values because of the largest $g$ factor. However, within the experimental error, there is no difference between the ee values in both the presence and absence of the magnetic field. For 2-PCO, although the ee values reach ca. 1-2\% ee, no MFE on ee is observed. The unbalanced ee between left- and right-CPL for 2PCHep is probably due to a peak overlap of the faster effluent enantiomer with a small amount of a photoproduct because ca. $1 \%$ ee was observed even upon LPL irradiation. Taking into account this value, the corrected ee values for 2-PCHep are ca. $1-2 \%$ ee for both right- and left-CPL, though the MFE was not obtained.

\section{Discussion}

Our kinetic simulation indicates that four reaction conditions are necessary for observing the MFE on asymmetric photolysis with CPL involving radical pair or biradical intermediates. These conditions are summarized as follows: (1) the presence of an escape process is indispensable, and the rate should be nearly equal to that of the intersystem crossing of radical pair or biradicals; (2) the recombination yields from singlet radical pair or biradical to each starting enantiomer must be sufficiently large; and (3) the rate of intersystem crossing of radical pairs or biradicals should be significantly changed upon applying magnetic fields. In addition, (4) a large Kuhn anisotropy $g$ factor at the irradiation wavelength is essential. These conditions are useful for exploring potential reaction systems.

Our kinetic simulation also covers two types of asymmetric photoreactions with CPL. One is an asymmetric photodestruction and the other is a photoderacemization..$^{4-6,8}$ In the case of $k_{\text {isc }}=0 \mathrm{~s}^{-1}$ or $\phi=0$ but $k_{\mathrm{y}} \neq 0 \mathrm{~s}^{-1}$ in the Eqs. 7 and 8 , the situation is reduced to the former type. We have confirmed that the variations of ee as a function of the sample decomposition simulated here precisely agree with predictions from the equation for asymmetric photodestruction with CPL, derived by Kagan et al. ${ }^{6}$ and Nakamura et al. ${ }^{8}$ On the other hand, in the limiting case where there are no photochemical side reactions, i.e. $k_{\mathrm{y}}=0 \mathrm{~s}^{-1}$ and $\phi=0.5$, only the latter type of reaction takes place. The ee change as a function of time is expected to approach an asymptotic value predicted by the equation ee $e_{\mathrm{pss}}$ $=g / 2$ in a photostationary-state. ${ }^{4,5}$

The photochemistry of 2-PCAs in the presence of molecular oxygen has been clarified, as shown in Scheme 3. This reaction scheme is consistent with the kinetic model described in Scheme 2. We have identified for the first time that the escape products from small-ring 2-PCAs are acetophenones and alk- 
Table 5. Enantiomeric Excesses (ee) of 2-PCAs Induced by $308 \mathrm{~nm}$ CPL Irradiation in the Presence and Absence of a Magnetic Field $(0.76 \mathrm{~T})$. Errors Correspond to Standard Deviations Derived from Five Independent Experiments. The Extent of Sample Decompositions Indicated in Brackets Are in the Range of 84-92\%

\begin{tabular}{lcccccccc}
\hline & \multicolumn{2}{c}{ Left-CPL/\% ee $($ decomp/\%) } & & \multicolumn{2}{c}{ Right-CPL/\% ee (decomp/\%) } & & \multicolumn{2}{c}{ LPL/\% ee (decomp/\%) } \\
\cline { 2 - 3 } 2-PCAs & $0 \mathrm{~T}$ & $0.76 \mathrm{~T}$ & & $0 \mathrm{~T}$ & $0.76 \mathrm{~T}$ & & $0 \mathrm{~T}$ & $0.76 \mathrm{~T}$ \\
\hline 2-PCH & $-2.6 \pm 0.2(91)$ & $-2.1 \pm 0.5(92)$ & & $+3.4 \pm 0.2(91)$ & $+3.3 \pm 0.4(92)$ & & $+0.3 \pm 0.3(92)$ & $+0.1 \pm 0.6(92)$ \\
2-PCHep $^{\ddagger}$ & $+2.2 \pm 0.5(89)$ & $+2.9 \pm 0.4(89)$ & & $-0.4 \pm 0.3(90)$ & $-0.1 \pm 0.5(88)$ & & $+1.5 \pm 0.5(91)$ & $+1.1 \pm 0.2(87)$ \\
2-PCO $^{\ddagger}$ & $+1.7 \pm 0.8(86)$ & $+0.9 \pm 0.4(91)$ & & $-1.6 \pm 0.5(86)$ & $-1.0 \pm 0.7(84)$ & & $+0.3 \pm 0.5(87)$ & $+0.5 \pm 0.6(88)$ \\
\hline
\end{tabular}

$\dagger$ The positive sign $(+)$ indicates excess for $R$ enantiomer of $2-\mathrm{PCH}$, ee $=100 \times([R]-[S]) /([R]+[S])$.

$\ddagger$ The positive sign $(+)$ indicates excess for the faster effluent enantiomer on a chiral-capirally GC.

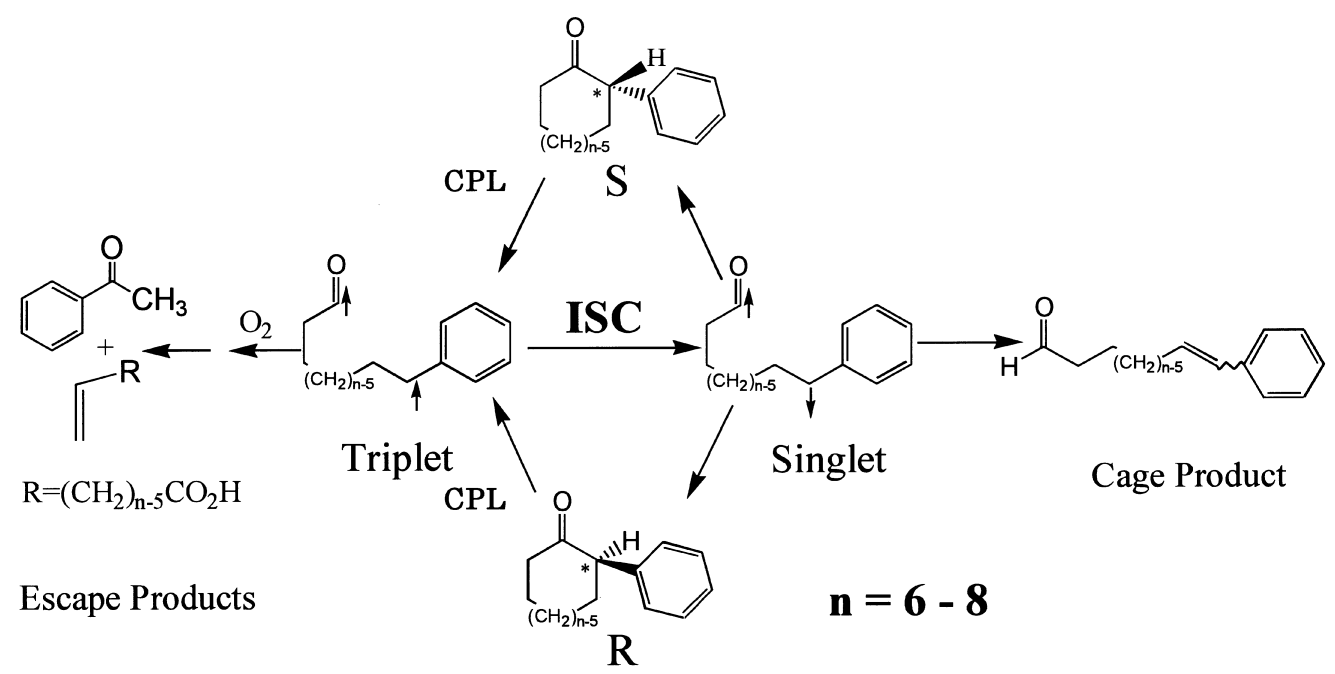

Scheme 3. Photolysis mechanism of 2-phenylcycloalkanones (2-PCAs, $n=6-8$ ) in the presence of molecular oxygen in solution.

enoic acids. This photoreaction may consist of two steps of photochemical reactions, as depicted in Scheme 4. First, the $\alpha$ photocleavage of 2-PCAs (Norrish type I) leads to the formation of triplet biradicals, and then results in the formation of Compound (A), as described in the brackets by a reaction with $\mathrm{O}_{2}$. Probably the bimolecular rate constant $\left(k_{\mathrm{esc}}\right)$ obtained from the transient absorption measurement indicates this reaction rate constant. Second, Compound (A) can be excited by the same UV light, and then subsequent intramolecular hydrogen abstraction (Norrish Type II) occurs to form acetophenone and the corresponding alkenoic acids. This reaction mechanism would also be supported from the facts that acetophenone is also produced in the Norrish Type II reaction of alkyl phenyl ketones, such as valerophenone and hexanophenone. ${ }^{46}$ The subsequent Norrish Type II reaction may occur so efficiently that Compound (A) is virtually unobservable in the present GC/MS analysis.

The second noticeable point of our findings concerning the photolysis of 2-PCAs is that the recombination yield from the singlet biradical to the starting 2-PCAs can be estimated by fitting procedures for the time evolution of the enantiomer concentration with LPL irradiation. Here, the recombination yields are estimated to be more than 0.8 for all 2-PCAs. However, these values are regarded as being rough estimations.

Although CPL-induced asymmetric photolysis of 2-PCAs $(n=6-8)$ was achieved to a few $\%$ ee the MFE on ee were not observed. The absence of the MFE can be rationalized based on four necessary conditions derived from our kinetic simula- tion. First, the escape rates from the triplet biradical in air-saturated solution are comparable to those of ISC for all 2-PCAs as shown in Table 3. Thus the condition (1) is fulfilled. Further, the recombination yields of the biradical are large enough to satisfy the condition (2) as listed in Table 4. Second, the anisotropy $g$ factors of 2-PCAs listed in Table 1 are comparable to the typical values for chiral carbonyl compounds as reported previously. ${ }^{5}$ Additionally, more than $1 \%$ ee of 2-PCAs after ca. $90 \%$ decomposition were obtained for all 2-PCAs ( $n$ $=6-8$ ) as expected. Consequently, conditions (1), (2), and (4) are fulfilled in this 2-PCAs reaction system. However, the ISC rates of acyl-benzyl biradicals $(n=6-8)$ were not affected by the static magnetic field in our experiments (below $8 \mathrm{~T}$ ). Therefore, the condition (3) is apparently not satisfied. This is the reason why we could not observe the MFE in this asymmetric photolysis.

The ISC process of acyl-benzyl biradical has been investigated in detail by Doubleday and Turro. ${ }^{26-28,30-33}$ They have claimed that spin-orbit coupling (SOC), which has no magnetic field dependence, plays a major role in the ISC process because of delocalization of the odd electron on the acyl oxygen. In general, it is recognized that if radical pairs are separated by $1 \mathrm{~nm}$ or more, the singlet-triplet energy gap is decreased and ISC is controlled by hyperfine coupling (HFC) induced nuclear-spin interactions, which are dependent on the magnetic fields and isotopes. ${ }^{11,17}$ However, since ISC of acyl-benzyl biradicals is dominated by the field-independent SOC, MFE on the ISC rate are diminished even for acyl-benzyl biradicals 


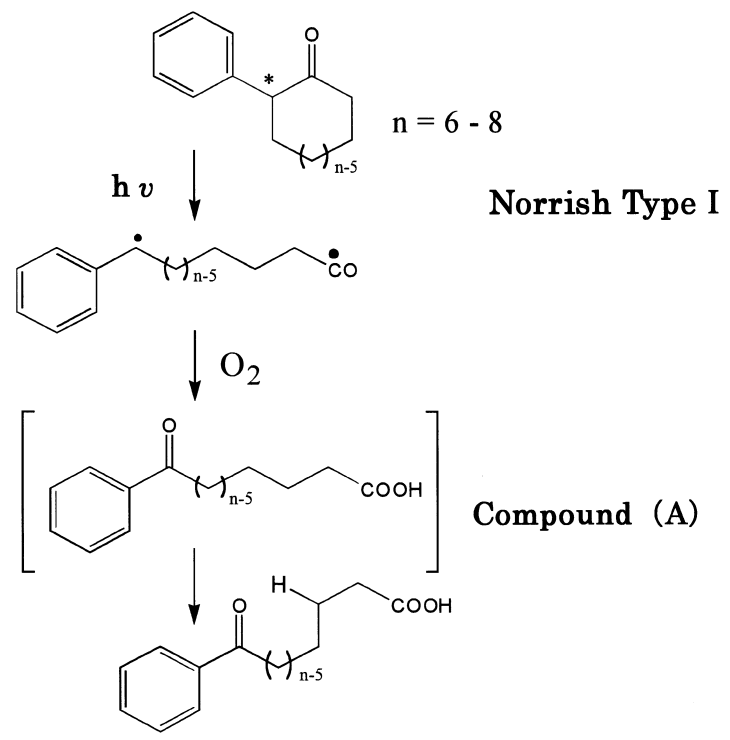

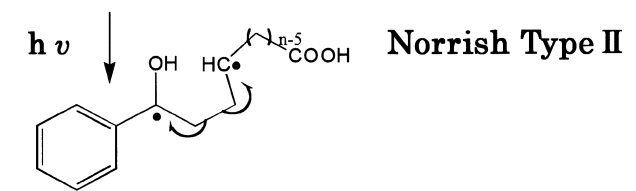<smiles>C=CCC(C)C(=O)O</smiles>

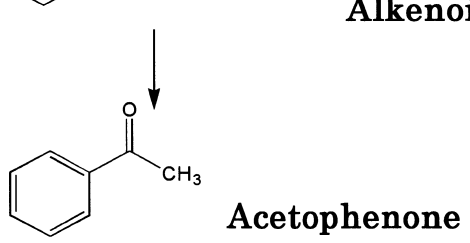

Scheme 4. An escape reaction mechanim of the triplet acylbenzyl biradicals with molecular oxygen.

with a long end-to-end distance $(n=10-12)$. According to studies by Doubleday and Turro, ${ }^{31,32}$ the SOC contribution to the ISC is estimated to be $76 \%$ for $n=12$ and $88 \%$ for $n=11$. Moreover, the SOC interaction will exponentially increase with decreasing radical pair distance so that contribution of magnetic-field-dependent HFC to the ISC becomes much smaller in short-chain biradicals. Consequently, ISC would be completely dominated by SOC in the acyl-benzyl biradicals derived from small rings $(n=6-8)$, and thus the MFE have not been observed.

CPL-induced ee changes of 2-PCH were simulated based on the assumption that $k_{\text {isc }}$ is decreased by one order of magnitude upon applying a magnetic field. Figure 7 shows two simulated curves. Line A indicates the actual ee changes based on our experimental values, where $k_{\text {isc }}$ is set to be the actural value of $1.5 \times 10^{7} \mathrm{~s}^{-1}$. In this case, the values of ee and the sample decomposition at $60 \mathrm{~min}$ reach to about $3 \%$ ee and $90 \%$, respectively. These values are in good agreement with the experimental values. (see Table 5). Line B is for the case where $k_{\text {isc }}$ is decreased by one order of magnitude $\left(1.5 \times 10^{6} \mathrm{~s}^{-1}\right)$, but all other parameters are fixed. It is apparent that an approximate

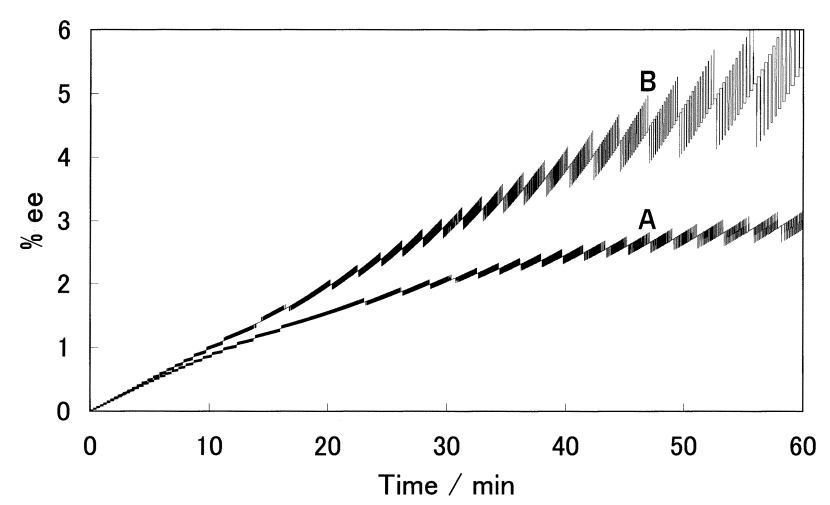

Fig. 7. Simulation results of the ee as a function of time. Parameters used here are as follows; Line A: $I_{0}: 5 \times 10^{-8}$ einsteins $\cdot \mathrm{cm}^{-2} \mathrm{~s}^{-1}, \eta: 0.30, \varepsilon_{R}: 37.15 \mathrm{M}^{-1} \mathrm{~cm}^{-1}, \varepsilon_{S}: 36.05 \mathrm{M}^{-1}$ $\mathrm{cm}^{-1}, k_{\text {isc }}: 1.5 \times 10^{7} \mathrm{~s}^{-1}, k_{\mathrm{y}}: 1.3 \times 10^{7} \mathrm{~s}^{-1}, \phi: 0.4$. Initial concentration for both $R$ and $S$ enantiomers is the same value of $2 \mathrm{mM}$. Line B: $k_{\text {isc }}: 1.5 \times 10^{6} \mathrm{~s}^{-1}$, other parameters are identical to those used for Line $\mathrm{A}$.

two-fold increase in the simulated ee is predicted if $k_{\text {isc }}$ could be decreased by one order of magnetude by applying a magnetic field. This difference may be distinguished in our chiral capillary GC experiments (see Table 5). It is not unusual that the ISC rate of biradical changes upon applying magnetic fields of more than one order of magnitude in the case where it is dominated by the HFC mechanism. ${ }^{12-15,17}$

In order to increase the MFE on asymmetric photolysis with CPL for a series of cycloalkanones, we can impose a restriction on the relative orientation of the acyl and alkyl radicals. Some of biradical intermediates derived from bicyclic ketones may not undergo a conformational change into an orientation favorable for SOC-induced recombination in the triplet state, as was pointed out by de Kanter and Kaptein. ${ }^{45}$ The HFC-induced ISC may become dominant for the biradical with specific stereochemistry, which suppresses ISC due to the SOC mechanism. Therefore, we still recognize the importance of the photochemical $\alpha$-cleavage of chiral cyclic ketones (Norrish type I), even if terminal acyl radicals are generated.

The authors are indebted to Mr. Jun Kojima (The University of Tokyo) who gave support in programming for the simulation. This work was supported by Sasagawa Scientific Research Grant from The Japan Science Society (No. 13-130).

\section{References}

1 W. A. Bonner, Orig. Life Evol. Biosphere, 21, 59 (1991).

2 M. Avalos, R. Babiano, P. Cintas, J. L. Jimenez, J. C. Palacios, and L. D. Barron, Chem. Rev., 98, 2391 (1998).

3 B. L. Feringa, and R. A. van Delden, Angew. Chem. Int. $E d .$, 38, 3418 (1999).

4 H. Rau, Chem. Rev., 83, 535 (1983).

5 Y. Inoue, Chem. Rev., 92741 (1992), and references cited therein.

6 G. Balavoine, A. Moradpour, and H. B. Kagan, J. Am. Chem. Soc., 96, 5152 (1974).

7 H. Nishino, A. Nakamura, and Y. Inoue, J. Chem. Soc., Perkin Trans. 2, 2001, 1693. 
8 A. Nakamura, H. Nishino, and Y. Inoue, J. Chem. Soc., Perkin Trans. 2, 2001, 1701.

9 H. Nishino, A. Nakamura, H. Shitomi, H. Onuki, and Y. Inoue, J. Chem. Soc., Perkin Trans. 2, 2001, 1706.

10 G. L. J. A. Rikken and E. Raupach, Nature, 405, 932 (2000).

11 U. E. Steiner and T. Ulrich, Chem. Rev., 89, 51 (1989).

12 R. Nakagaki, O. Takahira, and K. Hiruta, Chem. Phys. Lett., 233, 41 (1995).

13 R. Nakagaki, M. Yamaoka, O. Takahira, K. Hiruta, Y. Fujiwara, and Y. Tanimoto, J. Phys. Chem. A., 101, 556 (1997).

14 Y. Fujiwara, T. Aoki, T. Haino, Y. Fukazawa, Y. Tanimoto,

R. Nakagaki, O. Takahira, and M. Okazaki, J. Phys. Chem. A., 101, 6842 (1997).

15 R. Nakagaki, M. Yamaoka, and K. Mutai, Bull. Chem. Soc. Jpn., 72, 347 (1999).

16 I. Suzuki, R. Tanaka, A. Yamaguchi, S. Maki, H. Misawa, K. Tokumaru, R. Nakagaki, and H. Sakuragi, Bull. Chem. Soc. Jpn., 72, 103 (1999).

17 H. Hayashi, Y. Sakaguchi, and M. Wakasa, Bull. Chem. Soc. Jpn., 74, 773 (2001).

18 R. Nakagaki, Y. Tsujimoto, and K. Mutai, Chem. Phys. Lett., 244, 388 (1995).

19 R. Nakagaki and K. Mutai, Bull. Chem. Soc. Jpn., 69, 261 (1996).

20 Y. Konishi, P. Maruthamuthu, M. Okazaki, Y. Arakawa, and R. Nakagaki, Bull. Chem. Soc. Jpn., 72, 27 (1999).

21 G. Lem and N. J. Turro, Chem. Commun., 2000, 293.

22 R. A. Hegstrom and D. K. Kondepudi, Chem. Phys. Lett., 253, 322 (1996).

23 K. Soai, T. Shibata, and I. Sato, Acc. Chem. Res., 33, 382 (2000).

24 M. Avalos, R. Babiano, and J. C. Palacios, Chem. Commun., 2000, 887.

25 D. A. Lightner, in "Circular Dichroism," 2nd ed., ed by N. Berova, K. Nakanishi, and R. W. Woody, Wiley-VCH, New York (2000), Chap. 10.

26 C. Doubleday Jr., Chem. Phys. Lett., 64. 67 (1979).

27 N. J. Turro, K. C. Hwang, V. P. Rao, and C. Doubleday Jr., J. Phys. Chem., 95, 1872 (1991).
28 K. C. Hwang, N. J. Turro, and C. Doubleday Jr., J. Am. Chem. Soc., 113, 2850 (1991).

29 D. Weir, and J. C. Scaiano, Chem. Phys. Lett., 118. 526 (1985).

30 M. B. Zimmt, C. Doubleday Jr., I. R. Gould, and N. J. Turro, J. Am. Chem. Soc., 107, 6724 (1985).

31 M. B. Zimmt, C. Doubleday Jr., and N. J. Turro, J. Am. Chem. Soc., 107, 6726 (1985).

32 C. Doubleday Jr., N. J. Turro, and J. F. Wang, Acc. Chem. Res., 22, 199 (1989).

33 J. F. Wang, V. P. Rao, C. Doubleday Jr., and N. J. Turro, J. Phys. Chem., 94, 1144 (1990).

34 A. A. Baum, Tetrahedron Lett., 1972, 1817.

35 P. J. Wagner and T. J. Stratton, Tetrahedron, 37, 3317 (1981)

36 X. Lei, C. Doubleday Jr., and N. J. Turro, Tetrahedron Lett., 27, 467 (1986).

37 X. Lei, C. Doubleday Jr., M. B. Zimmt, and N. J. Turro, J. Am. Chem. Soc., 108, 2444 (1986).

38 M. A. Miranda, E. F. Sanchis, and J. P. Prieto, J. Org. Chem., 64, 3802 (1999).

39 R. Hayano, and T. Takahashi, "Computer in Physics," Kyoritsu Shuppan (1992). Simulation source program was written in the $\mathrm{C}$ programming language.

40 J. H. Ryan, and P. J. Stang, Tetrahedron Lett., 38, 5061 (1997).

41 G. Berti, B. Macchia, F. Macchia, and L. Monti, J. Chem. Soc. C, 1971, 3371 .

42 K. Ishihara, M. Kaneeda, and H. Yamamoto, J. Am. Chem. Soc., 116, 11179 (1994).

43 J. N.Murrel, in "The Theory of the Electronic Spectra of Organic Molecules," Chapman and Hall (1963), Chap. 8.

44 S. L. Murov, I. Carmichael, and G. L. Hug, in "Handbook of Photochemistry," 2nd. ed., Revised and Expanded, Marcel Dekker (1993), Section 12-3.

45 F. J. J. de Kanter and R. Kaptein, J. Am. Chem. Soc., 104, 4759 (1982)

46 N. J. Turro, in "Modern Molecular Photochemistry," Benjamin/Cummings, Menlo Park (1978), Chap. 10. 